\title{
A cien años de la reforma de Córdoba, 1918-2018 La época, los acontecimientos, el legado*
}

\author{
Álvaro Acevedo Tarazona ${ }^{\star \star}$
}

\begin{abstract}
Resumen:
La autonomía y el cogobierno son las conquistas más representativas de la Reforma de Córdoba de 1918, una proclama estudiantil del orden político y académico que traspasó las fronteras nacionales del continente latinoamericano. Si bien los acontecimientos y consecuencias de esta Reforma han llamado la atención de especialistas en la historia de la universidad y de los movimientos sociales, a casi cien años del legado de Córdoba es más que necesario hacer una valoración retrospectiva desde la universidad actual hasta los principios esenciales del Manifiesto de 1918, los ideales y las tareas de la universidad.
\end{abstract}

Palabras claves: Manifiesto, Córdoba, Universidad, Reforma, Estudiantes, América Latina.

\section{Abstract:}

Autonomy and co-governance are the most representative achievements of the 1918 Cordoba Reform, a student claims of political and academic who has gone national boundaries of Latin America. While the events and consequences of this reform have drawn the attention of specialists in school history and social movements, almost one hundred years the legacy of Córdoba is more than necessary to make a retrospective evaluation from the university today to the early Essential Manifest of 1918, the ideals and the tasks of the university.

Keywords: Manifest, Cordoba University, Reform, Students, Latin America.

La Reforma de Córdoba dejó una impronta perdurable en la historia política y de la cultura de Argentina. Los acontecimientos de Córdoba fueron el triunfo de un movimiento estudiantil que supo aprovechar la coyuntura social en un país marcado por la permanencia de un sistema autoritario de viejas costumbres. La importancia de las universidades argentinas y su papel organizador político y social de las clases medias fue creciendo a través de los años, siempre coincidiendo con las inestabilidades políticas. Se ha propuesto incluso una interesante periodización de la historia de las universidades argentinas demarcada por los altibajos políticos (SIGAL, 2002, p. 21) ${ }^{1}$. Para entender la importancia y

\footnotetext{
*Artículo Tipo 2: de reflexión según Colciencias.

** Doctor en Historia, Profesor de la Universidad Industrial de Santander. Director del grupo de investigación Políticas, Sociabilidades y Representaciones Histórico-Educativas (PSORHE). E-mail: tarazona20@gmail.com.

${ }^{1}$ Esto se evidencia con los desórdenes políticos originados por los golpes de Estado de 1930, 1943, 1955, 1966 y 1976.
} 
trascendencia de esta Reforma es necesario examinar de manera sucinta el contexto social y político de Argentina a comienzos del siglo XX y los acontecimientos de 1918.

\section{La época}

Luego de finalizada la Primera Guerra Mundial hubo nuevas corrientes de pensamiento que influyeron en una generación que veía cómo las potencias no sólo se repartían entre ellas los mercados mundiales sino también sus triunfos militares. En esta lucha fueron más evidentes sobre América Latina las intenciones expansionistas de Estados Unidos. Voces de políticos e intelectuales en el continente denunciaron este "imperialismo yanqui" con su reafirmación de la Doctrina Monroe, "América para los americanos", proclamada desde el año de 1823 por John Quincy Adams y James Monroe ${ }^{2}$. En este mismo contexto, la Revolución Soviética mostraba que era posible el alzamiento de las masas obreras para promover en el corto plazo los cambios que las sociedades tradicionales demandaban.

Advierten especialistas que en el movimiento de Córdoba hubo influencias de las ilusiones demoliberales y pacifistas de la corriente wilsoniana, muy en boga entre la juventud por los años de 1918 y 1919. Una influencia que mostraba el advenimiento del "espíritu nuevo": la victoria del idealismo novecentista sobre el positivismo del siglo XIX. En este orden de influencias, el neoideal bergsoniano también prepararía el Movimiento de la Reforma, sin descontar la inspiración de una raíz burguesa y anticlerical. De ahí el carácter social de la Reforma. Si bien podría señalarse la circulación de estas ideas y hasta el triunfo sobre el positivismo, no es posible pasar por alto que las exigencias de los estudiantes por una transformación académica situaban como vanguardia los discursos de las ciencias y tecnologías, provenientes de ese mismo positivismo que tanto se criticaba (Acevedo Tarazona, 2006, p. 183-218). Tampoco hay que pasar por alto que mientras en Europa se daban pasos decisivos en el avance de disciplinas como la medicina, la matemática, la paleontología y la geología, en América del Sur y particularmente en Argentina los estudios científicos eran casi inexistentes (Vera, citado en Soto Arango; Lucena \& Rincón, p. 183-218). A mediados del siglo XIX en la Universidad de Córdoba sólo se estudiaba teología y derecho. Posteriormente, en 1868, se introdujeron las matemáticas y la medicina. Este letargo académico y disciplinario iba de la mano con tarea civilizadora asignada al maestro, forjador de la nación y modelo de autoridad y virtudes (Acevedo Tarazona, 2008, p. 61-82). "La rebeldía estalla ahora en Córdoba y es violenta porque aquí los tiranos se habían ensoberbecido y era necesario borrar para siempre el recuerdo de los contrarrevolucionarios de Mayo", señaló precisamente el Manifiesto de los estudiantes para luchar contra las tradiciones anacrónicas, el

\footnotetext{
${ }^{2}$ Estados Unidos reafirmó la política de intervención en el continente latinoamericano mediante la Doctrina del Destino Manifiesto, proclamada por Theodore Roosvelt en 1904. Esta doctrina era una especie de carta blanca para la intervención de los Estados Unidos en América Latina y el Caribe si cualquier nación bajo influencia de la órbita estadounidense dejaba en peligro los derechos o propiedades de sus empresas.
} 
"derecho divino" de los profesores y para reclamar un gobierno democrático en la universidad.

Ahora bien, si el Movimiento Reformista de Córdoba fue consecuencia de influencias externas, sus principales causas hay que encontrarlas en la nueva e innegable situación social y política en la Argentina de aquella época.

\section{El contexto social y político}

La Universidad de Córdoba junto a las universidades de Buenos Aires y Nacional de La Plata eran las de mayor tradición en Argentina. Centros de educación superior en los que predominaba la rutina pedagógica y la ortodoxia católica. El fin de la Primera Guerra Mundial, el quiebre de ideales que implicó el cambio de siglo, la Revolución Rusa y el acceso al poder del Partido Radical argentino en 1916 mediante el primer sufragio universal fueron acontecimientos que repercutirían en el movimiento estudiantil (Sigal, 2002, p. 79). No se puede tampoco pasar por alto que la urbanización era un hecho irreversible en la Argentina de aquella época, sumado a los contingentes de obreros que entraban a desempeñar un papel de primer orden en la economía.

Para Luis Marco del Pont (Tünnermann, 1983, p. 31) y otros especialistas como Renate Marsiske (1999) hubo unas causas nacionales de este acontecimiento estrechamente vinculadas a la clase media emergente. Para esta época la población de Argentina aumentaba vertiginosamente y en 1895 las ciudades albergaban al $60 \%$ de la población (Del Pont, 2005). La nueva clase media urbanizada fue el principal actor del movimiento reformista que intentaría a cualquier costo lograr acceder a la universidad, un espacio de privilegios que durante mucho tiempo había sido dirigido por la tradicional oligarquía terrateniente y el clero.

Visto así, el ambiente social que se vivía en Argentina a comienzos del siglo XX preparaba el camino para los cambios que se aproximaban. La creciente masa de inmigrantes que llegaba a territorio argentino con ideas socialistas y liberales comenzó a establecer los sindicatos y las federaciones obreras. Su impacto era tan grande que el gobierno del general Julio Argentino Roca debió sancionar la Ley de Residencia para extranjeros, la cual dio autoridad al Poder Ejecutivo para impedir la entrada o expulsar del país a cualquier extranjero cuya conducta comprometiera la seguridad nacional o perturbara el orden público. La presión de los inmigrantes, de los obreros y de las clases medias emergentes por vincularse en el aparato productivo y acceder a la movilidad social no iba a esperar. 1917 fue el año con mayor número de huelgas obreras; según estimaciones del Departamento Nacional del Trabajo se presentaron 138 huelgas, las que agruparon a un total de 136.002 personas, para entonces casi la población entera de Córdoba. Las huelgas no sólo se dieron en Córdoba, todo el país se hallaba inmerso en una ola de manifestaciones sin precedentes. Sin embargo, Córdoba sería el escenario de la gran ola de manifestaciones y conflictos obreros. Una de sus estrategias para ser escuchados consistió en apoyar a los estudiantes en sus demandas de transformación universitaria. No obstante, los dirigentes sindicales 
debieron interceder ante sus compañeros de lucha para que apoyaran a esta nueva generación de estudiantes que ya no tenía estrechos vínculos con aquellas familias distinguidas de la sociedad argentina.

Consecuencia de esta ebullición política, las ideas socialistas habían incursionado profundamente en los universitarios y en los dirigentes obreros. El Partido Socialista de Argentina era uno de los más fuertes de América Latina y daría su apoyo incondicional al movimiento estudiantil reformista con el primer diputado socialista del continente: Alfredo Palacios. El líder socialista se mostró como uno de los líderes incondicionales del movimiento; el mismo que a su vez sería visto como un auténtico maestro por parte de los estudiantes. El movimiento también recibiría el respaldo del Partido Socialista Internacional, muy a propósito de lo expresado por La Internacional: la influencia clerical en Córdoba va decayendo a pasos agigantados y la loable actitud de los estudiantes que protestan contra el predominio de esa influencia nefasta en la enseñanza es una demostración evidente que remarcan con simpatía los socialistas internacionales.

Cabe destacar que no sólo los acontecimientos políticos y sociales inmediatos a Córdoba fueron los desencadenantes del movimiento reformista. Desde comienzos del siglo XX era inevitable el cambio social en Argentina. La región conocida como la Pampa Húmeda continuaba su colonización y crecían centros industriales en Buenos Aires, Córdoba y Santa Fe. Alrededor de esta dinámica comenzaron a originarse particulares grupos sociales que se mostraban adversos a la tradición de una "casta social" constituida principalmente por "el laboreo feudal de la tierra y una oligarquía terrateniente hegemónica en el poder por prácticas políticas medievales" (Ceballos, 1986, p. 42). Justamente con Hipólito Yrigoyen a la cabeza, el radicalismo fue el canalizador de los nuevos grupos sociales que llegarían al poder por primera vez mediante el establecimiento del sufragio universal. Para el Partido Radical la universidad era el último reducto de la oposición, de ahí que apoyase los programas de cambio de los estudiantes universitarios.

Luego de fracasadas las revoluciones de 1890, 1893 y 1905 que exigían libertad electoral, apareció el Manifiesto de la Unión Cívica Radical en el que se expresaba su radicalismo como una fuerza "cumplidora" de la constitución con el propósito de "reparar" el orden preexistente (Cantón, Moreno \& Ciria, 2005, p. 14). No sería irrelevante analizar entonces las características del Partido Radical que estaba en el poder mientras se gestaba el movimiento reformista de Córdoba.

\section{La Unión Cívica Radical}

El Partido Radical tomó el poder por la vía de las urnas y no por la vía revolucionaria, lo que ya presagiaba su incapacidad para hacerse al respaldo necesario en aras de mantenerse en el poder. Este triunfo electoral fue resultado de una elección relativamente reñida, lo que dejó en entredicho la correcta puesta en marcha de su propuesta política. El partido y su máximo representante Hipólito Yrigoyen estarían obligados a maniobrar cautelosamente para ir ganando favorabilidad. 
El voto universal obligatorio implementado por Roque Sáenz Peña fue decisivo para el triunfo del radicalismo en 1916, pero tan sólo en un comienzo porque debían desmontarse todas las antiguas estructuras estatales, lo cual no era fácil para el radicalismo puesto que eran mayoría en la Cámara pero no en el Senado. De otro lado, el gobierno no encontró un respaldo unificado de la población, lo que posteriormente sería determinante para el golpe de 1930. No está demás señalar que si bien el gobierno radical se mostró a favor de los estudiantes, finalmente éste no le daría un respaldo absoluto al movimiento de la Reforma Universitaria (Cantón et al. 2005, p. 15). La prensa de mayores recursos económicos, prestigio y capacidad de difusión dejó de apoyar a Yrigoyen, y al poco tiempo de comenzar su primer gobierno ya era su más fuerte opositora. Se diría pues que Yrigoyen fue un gobernante elegido por una mayoría popular, pero con una fuerte oposición de los partidos y una carencia de apoyo de "organizaciones o factores de poder que contaran con medios regulares de acción o expresión" (Cantón et al. 2005, p. 17). En el mismo partido radical existía una notable oposición hacia Yrigoyen, sobre todo de la facción intelectual del partido que daría su apoyo a Máximo Marcelo Torcuato de Alvear. El propósito de estos últimos era formar un partido alterno a espaldas de Yrigoyen. De manera que la mayor parte de lo realizado en el gobierno parecía ser obra exclusiva del carisma de su máximo dirigente.

El diario La Voz del Interior apoyó el movimiento estudiantil de Córdoba. Sus propios directores formarían parte activa de la Reforma Universitaria, como el caso de Raúl Silvestre Remonda que sería presidente del Centro de Estudiantes de Derecho y presidente de la Federación Universitaria de Córdoba (Cantón et al. 2005, p. 33). La Voz del Interior desempeñó un papel excepcional en el triunfo de la Reforma con su carácter idealista, romántico y revolucionario. El diario criticaba los privilegios de las pocas manos que dirigían la Universidad, además exigía nuevos planes de estudio, asignaturas y en general cambios positivos para el nuevo escenario mundial.

\section{El estallido de 1918}

Desde el siglo XVIII la Universidad de Córdoba permanecía prácticamente inmóvil y con una fuerte influencia clerical. En la biblioteca era imposible encontrar algún libro sobre Bernard, Stammler, Darwin, Haeckel, Marx o Engels; el escudo de la universidad llevaba grabado el nombre de Jesús y se festejaba el 8 de diciembre, día de la Virgen de la Concepción; así mismo, el juramento profesional se prestaba obligatoriamente sobre los Santos Evangelios (Ciria \& Sanguinetti, 1987, pp. 23, 25). En la universidad era también notable la influencia de la "Corda Frates", según lo expresado por la La Voz del Interior:

(...) entidad jesuita, vive al margen de los partidos y las tendencias, pretende mejorar la política de Córdoba y a veces lo consigue, se infiltra por todas partes, descomponiendo y perturbando el organismo social... para ella las banderías son pretextos accidentales; milita en las esferas del poder succionando los jugos del presupuesto y extiende los tentáculos de la intriga en busca de ventajas probables. Nada significa la posición partidaria, el logro 
de ambiciones es la finalidad permanente que mueve sus resortes ocultos" ( $\mathrm{La}$ Voz del Interior, 2 de abril de 1919).

Las huelgas de las facultades de Derecho en 1903 y Medicina en 1905 fueron los antecedentes inmediatos de la Reforma Universitaria. El detonante de los hechos de Córdoba fue la suspensión del Hospital de Clínicas en el mes de diciembre de 1917, dependiente de esta universidad. En 1918 los estudiantes de estas facultades se declararon en huelga y el descontento fue creciendo hasta que en abril de este año se creó la Federación Universitaria Argentina, conformada por los estudiantes de Tucumán, Santa Fé, Córdoba, La Plata y Buenos Aires. José Nicolás Matienzo fue designado por el gobierno de Yrigoyen como interventor de la universidad. Éste inmediatamente reformó los estatutos y llevó a cabo la elección de autoridades el 15 de junio. Los estudiantes consideraron que la elección había sido trucada por los jesuitas -el poder de facto en la universidad-y no la aceptaron (Cantón et al. 2005, p. 78). El 21 de junio de 1918 difundieron el Manifiesto Liminar (La juventud argentina de Córdoba a los hombres libres de Sud América), previa declaratoria de huelga indefinida.

El inicio de los acontecimientos, que condujeron a la elaboración del Manifiesto Liminar, puede establecerse a fines de 1917 cuando los Estudiantes de Medicina rechazaron la supresión del internado en el Hospital Nacional de Clínicas. Al no ser atendidas sus reclamaciones se constituyó el Comité pro Reforma que posteriormente daría paso a la Federación Universitaria de Córdoba. El 20 de marzo el Consejo Superior de la Universidad decidió no tomar en cuenta ninguna solicitud de los estudiantes. Estos reaccionaron violentamente. El Secretario de la Universidad solicitó la presencia de la policía para dar inicio a las clases. En víspera de la inauguración de los cursos, el Comité pro Reforma decidió declarar la huelga general.

El 2 de abril el Consejo Superior, aduciendo reiterados actos de indisciplina por parte de los estudiantes que perturbaban el orden en la institución, expidió un comunicado en el que impedía la entrada de cualquier persona diferente a profesores o empleados de la universidad. En vista de las circunstancias y como última medida, los integrantes del Comité pro Reforma pidieron la intervención del Ministro de Instrucción Pública en la universidad. Los estudiantes lograron su cometido y el 11 de abril el presidente Yrigoyen envió al procurador general de la Nación, José Nicolás Matienzo, mientras en Buenos Aires era oficialmente fundada la Federación Universitaria Argentina (FUA), conformada por los dirigentes estudiantiles de diversas universidades del territorio argentino.

En la inspección de Matienzo fueron comprobadas algunas irregularidades señaladas por los estudiantes. En razón de este clima de agitación, el funcionario decidió presentar un proyecto de reformas al estatuto para "democratizarlo". Las reformas decretadas por Yrigoyen el 7 de mayo otorgaron participación por primera vez al profesorado en la elección de los directivos de la universidad (rector, decanos, vicedecanos). Mientras tanto el 16 de mayo el Comité pro Reforma dejó de existir para dar paso a la Federación Universitaria de Córdoba 
(FUC), dirigida por Enrique Barros, Horacio Valdés e Ismael Bordabhére (La Gaceta Universitaria, 2009, p. 93). A la par se crearon grupos anti reformistas como el Comité pro Defensa de la Universidad y los Centros Católicos de Estudiantes. También se presentó una renuncia masiva de profesores que se oponían a las reformas.

El 28 de mayo se dio por primera vez en la historia de la Universidad de Córdoba la elección de decanos, vicedecanos y consejos directivos. La victoria de los candidatos de la FUC era notable (La Gaceta Universitaria, 2009, p. 113), pero aún faltaba la elección del cargo más importante: el rector. La FUC apoyó al candidato Enrique Martínez Paz, mientras que la "Corda Frates" tenía como candidato a Antonio Nores. Tras dos votaciones fallidas por no obtener ninguno de los candidatos una mayoría notable, se llevó a cabo una tercera votación en la que Antonio Nores resultó ganador con 24 votos, frente a los 14 de Martínez Paz. En cuestión de minutos los estudiantes invadieron el recinto desalojando a los policías allí presentes. Dos días después, Nores intentó asumir la rectoría. El ánimo de los estudiantes se enervó y reaccionaron con violencia. Tres delegados de la FUC se reunieron en el despacho de Nores para pedirle la dimisión al cargo de rector. Éste respondió a sus peticiones declarando: "prefiero antes de renunciar que quede el tendal de cadáveres de los estudiantes" (Ciria, \& Sanguinetti, p. 29). El conflicto ya adquiría proporciones nacionales. A los estudiantes cordobeses se unió no sólo la FUBA (Federación Universitaria de Buenos Aires) y las demás federaciones universitarias, sino también los sindicatos de trabajadores. Así, la ciudad de Córdoba comenzó a ser escenario de diversas manifestaciones.

El movimiento reformista expresó sus intenciones en el Manifiesto Liminar de la Reforma Universitaria de Córdoba redactado por Deodoro Roca el 21 de junio de 1918 y firmado por los estudiantes miembros de dicha organización (La Gaceta Universitaria 1918-1919, p. 143). En el documento reclamaron la defensa de un gobierno universitario democrático y que este derecho de gobernabilidad no proviniera del autoritarismo. Consideraron también que la relación entre gobernantes y gobernados resultaría perjudicial y generaría constantes trastornos sino partía de una vinculación espiritual.

Entre el 20 y el 31 de julio de 1918 se llevó a cabo en Córdoba el Primer Congreso Nacional de Estudiantes, convocado por la FUA e integrado por las federaciones de Buenos Aires, Córdoba, La Plata, Tucumán y Litoral (La Gaceta Universitaria 1918-1919, p. 148). En dicho Congreso fueron discutidos novedosos esquemas de leyes y estatutos sobre autonomía universitaria, gobierno tripartito paritario, asistencia libre, docencia libre, bienestar estudiantil, libertad de juramento y nacionalización de las universidades provinciales del Litoral y Tucumán. Allí también fue declarado el 15 de junio como el día de La Reforma (La Gaceta Universitaria 1918-1919, pp. 155-159).

Como el rector elegido Antonio Nores mantenía cerrada la institución, los reformistas radicales exigieron al presidente Yrigoyen la presencia de un nuevo interventor en la universidad. Sus peticiones fueron atendidas y el 2 de agosto fue 
nombrando en este cargo el médico y abogado Telémaco Susini, un representante anticlerical que en sus años de juventud, siendo estudiante universitario, participó activamente en el incendio del colegio de jesuitas El Salvador. Susini ya había respaldado abiertamente a la Federación Universitaria. Esto evidentemente despertó el pánico en el sector clerical de la universidad y el 7 de agosto Nores renunció a su cargo ante la Asamblea Universitaria alegando la actitud del gobierno al nombrar el nuevo interventor. Susini jamás arribó a Córdoba, lo que generó nuevos actos de manifestaciones por parte de los estudiantes. El gobierno nombró un nuevo interventor: el propio Ministro de Instrucción Pública, José Salinas. Contrario de lo que se esperaba, no hubo ninguna acción por parte de este funcionario, lo que generó otra ola de protestas que logró reunir a unas 20.000 personas lanzando consignas contra el clero. En vista de las circunstancias, los estudiantes decidieron actuar. El 9 de septiembre un grupo de alrededor de cien estudiantes ingresó y se apoderó de la universidad mientras llegaba el ministro. Transcurridas unas horas de ocupación, la fuerza pública ingresó a la universidad. Varios estudiantes fueron encarcelados y se les abrió un proceso por sedición. Pese a estas acciones de fuerza y represión de la fuerza pública el movimiento cumplió su objetivo. En horas de la tarde se comunicó el arribo del ministro. Rápidamente el proceso judicial de los estudiantes fue olvidado.

Finalmente, el ministro hizo presencia en la universidad recibiendo inmediatamente la renuncia de todos los académicos y de muchos profesores a su cátedra. Se reajustaron las administraciones y la docencia. Se reabrió el internado del Hospital de Clínicas, se comprobaron anomalías financieras y muchos reformistas, entre ellos Deodoro Roca, ocuparon las nuevas cátedras. Fueron también reorganizados los consejos y fue designado el doctor Eliseo Soaje como nuevo rector. El triunfo fue total.

\section{La Reforma}

El movimiento estudiantil de Córdoba buscaba lograr una participación plena del estudiante como ciudadano de la república universitaria, capaz de elegir y ser elegido. También suprimir el dogmatismo imperante mediante el establecimiento de la docencia libre para asegurar la existencia de cátedras paralelas o nuevas. La Reforma pretendía plantear temas de proyección latinoamericana en el que participaran los obreros y público en general no matriculado formalmente en la universidad. Así mismo, los estudiantes denunciaban el atraso científico de la universidad y sobre todo el carácter "arcaico y elitista del sistema de gobierno en la institución" (Buchbinder, 2005, p. 95).

El manifiesto rechazó la reforma liberal promulgada a la Universidad de Córdoba por José Nicolás Matienzo y apoyada por el clero, pues consideraba que lo único que hacía era mantener el dominio de "una casta de profesores". Así pues, reclamó el derecho de la insurrección desconociendo el proceso electoral llevado a cabo en el mes de junio de 1918, y del que se dijo que hubo irregularidades porque no se firmó el acta del nombramiento oficial del nuevo rector. Los estudiantes aseguraron haberse levantado contra un régimen administrativo 
autoritario en el que las funciones públicas se ejecutaban favoreciendo a determinados grupos. La Universidad de Córdoba, por demás, expresaron los estudiantes, era una institución donde no se reformaban estatutos por miedo a represalias y se pagaban favores, sin descontar que la práctica docente estaba dogmatizada y muy apartada de las ciencias modernas. El Manifiesto hizo llamado a todos los estudiantes del continente a liberarse de ese autoritarismo universitario imperante en todo el territorio y exigir que sus ideas fuesen reconocidas por medio de sus representantes (Manifiesto Liminar de la Reforma Universitaria, 2009). Estos fueron algunos de los puntos fundamentales consignados en el documento de 1918:

- Autonomía universitaria. Sin duda el punto más polémico y trascendental de la Reforma que sostenía que la universidad debía ser autogobernada, eligiendo sus propias directivas y formulando sus propios estatutos y programas de estudio. El propósito era que los asuntos políticos externos no influyesen en el ritmo de la actividad universitaria. Dicho principio también implicaba que la fuerza pública no ingresara a los recintos universitarios. De alguna manera también proponía una autarquía financiera, un fin hoy imposible de cumplir.

- Cogobierno. Otro de los puntos más importantes de la Reforma, el cual implicaba que los profesores, estudiantes y egresados participasen en el gobierno universitario, práctica que se extendió a lo largo de los continentes americano y europeo.

- Libertad de cátedra o docencia libre con cátedras paralelas y cátedras libres. Con esto se buscaba garantizar que existiese la mayor cantidad posible de corrientes de pensamiento y tendencias sin censuras ni prejuicios. Cualquier cátedra tenía autonomía para investigar y enseñar y no podía ser vigilada académicamente. Así mismo, debía haber variedad de cátedras que podrían ser elegidas libremente por el estudiante.

- Libertad académica para el análisis y expresión de ideas filosóficas, científicas, sociales y políticas.

- Misión social de la universidad para que su función social fuese mucho más allá de la simple enseñanza de las aulas de clase. La educación universitaria debía involucrarse en la investigación y la solución de los problemas de la sociedad y de las naciones. Lo que hoy se llama la extensión universitaria.

- Vinculación de la universidad con el resto del sistema educativo nacional de base. Esto implicaba el apoyo universitario en los procesos de formación y una real cohesión del sistema de Educación Superior con los niveles medio general, técnico y primario.

- Asistencia libre a clases para facilitar el proceso académico a los estudiantes de clase media que tuviesen que desempeñarse como trabajadores.

- Docencia libre, es decir, el aula disponible para todo aquel que quisiera impartir sus conocimientos sin importar su corriente de pensamiento. Esto 
unido a concursos de oposición para seleccionar el profesorado y periodicidad de las cátedras.

- Gratuidad de la enseñanza superior para que la educación superior fuese asequible a todos los sectores sociales.

- Unidad latinoamericana, lucha contra cualquier forma autoritaria de gobierno.

En síntesis, tres fueron los puntos esenciales de la Reforma de Córdoba: autonomía y gobierno universitario, cambios en la enseñanza y métodos docentes y proyección política y social de la universidad (Tünnermann, 2008). Lo más importante para destacar fue el alcance latinoamericano del movimiento. Pronto éste dejó de ser una reivindicación local que demandaba la reapertura del Hospital de Clínicas para constituirse en un movimiento continental que planteaba la construcción de una nueva universidad. Como lo señala Luis Marco del Pont: "De un movimiento provinciano, de un barrio, el Clínicas, pasó a tomar la ciudad de Córdoba, después el país y más tarde retumba por todas las universidades del continente americano" (Del Pont, 2005, p. 176).

A partir de esfuerzos participativos que reclamaban para la comunidad estudiantil un derrotero distinto a la formación tradicional, marcada bajo la impronta de la Iglesia, los poderes civiles y la influencia de la clase dominante, el movimiento estudiantil de 1918 significó un primer intento por redefinir el curso de una universidad. No hay que olvidar que la universidad del siglo XIX en Latinoamérica se configuró bajo la herencia de un modelo que tuvo en la Iglesia Católica y la universidad napoleónica a sus principales referentes que sellaron, hasta ese entonces, una política vertical que dejaba por fuera de toda posibilidad de participación a profesores y estudiantes en el discurso de orientación del verdadero sentido de la universidad pública, dependiente en grado sumo del poder estatal o eclesiástico con afectación de la autonomía académica. Los jóvenes de Córdoba reclamaban que la Iglesia y los poderes civiles sacaran las manos de la universidad, exigían democracia y pedían que la universidad tuviera una clara función social. En el fondo planteaban que debía terminar la era oscura e intranscendente de la universidad aristocrática republicana.

\section{A modo de cierre. El legado de Córdoba}

Los cambios propuestos por la Reforma Universitaria de Córdoba consolidaron su papel como defensora de una educación superior al servicio de la sociedad. El espíritu reformador se extendió por todo el continente americano. Las primeras ciudades contagiadas serían La Plata y Buenos Aires que incorporaron el cogobierno estudiantil. Las de Tucumán y El Litoral fueron nacionalizadas como lo exigía la FUA. Para 1921 el programa reformista se había puesto en marcha en todas las universidades del país, con puntos tan importantes como la participación estudiantil en el gobierno universitario, la docencia libre, la libre asistencia a clase, la extensión universitaria, entre otros. Cabe señalar que fue entre 1918 y 1921 el periodo de máxima gloria de la Reforma, justamente mientras Yrigoyen estaba al poder (Del Pont, 2005, p. 179). 
En América Latina el movimiento tuvo gran impacto. Desde el comienzo los reformistas cordobeses tuvieron una visión continental, tal como se puede evidenciar en el encabezado del manifiesto: "La juventud argentina de Córdoba a los hombres libres de Sudamérica". Para la difusión del Manifiesto en toda América Latina fue fundamental el Primer Congreso Internacional de Estudiantes de la Reforma celebrado en México en 1921. La reforma llegó en 1919 a Perú (donde sentaría sus bases un partido político, el APRA), en 1920 a Chile, en 1921 a México, en 1922 a Colombia, en 1923 a Cuba, en 1927 a Paraguay, en 1928 a Brasil y Bolivia y en 1933 a Costa Rica.

A casi cien años del Manifiesto hay que rescatar las ideas de la Reforma y sus conquistas, entre ellas el sentido social y político del movimiento, la democratización y la autonomía universitaria, las cátedras libres y por concurso, la participación estudiantil en el gobierno universitario y la dimensión latinoamericana. No se trataba sólo de introducir cambios internos en la universidad. La reivindicación de derechos por parte de los estudiantes de la Reforma de 1918 también era la lucha de los universitarios frente a un enemigo que venía dando pasos de gigante en el orbe: el imperialismo. Era la lucha por lograr la cristalización de un nuevo modelo de universidad que se ajustara a las demandas del contexto social; una apuesta donde predominara la creación de conocimientos y no su mera transmisión y gerencia con el único afán formar de una delgada capa de capital humano altamente disciplinado para ingresar al mercado (Aboites, 2008, p. 12).

En el movimiento estudiantil de 1918 desempeñaría un papel importante la clase media. Precisamente serían los hijos de esta clase medial quienes iban a reclamar una educación superior acorde a las exigencias de la modernización económica del país. Su propósito era acceder a capas sociales antes vetadas y a profesiones liberales restringidas. No obstante, la Reforma de Córdoba no logró cambios sustanciales en la sociedad argentina ni rompió el modelo de universidad napoleónica decimonónico que tanto criticaba. Es por esto que los críticos del movimiento estudiantil de Córdoba ven en la Reforma una profunda frustración y sólo un interés de movilidad social por parte de las clases medias. Pese a esta crítica, después de Córdoba la universidad dejó de ser eminentemente elitista y clerical, como si lo había sido la universidad colonial y en parte la republicana, esto era, virreinatos del espíritu, academias señoriales (Tünnermann, 1918-2008, p. 39). Los estudiantes de Córdoba expulsaron del claustro a la Compañía de Jesús y promovieron un proyecto nacional de universidad. Por este triunfo y propósito los estudiantes fueron exitosos (Aboites, 2010). A partir de ese momento, diría Germán Arciniegas, la universidad latinoamericana "no fue lo que ha de ser, pero dejó de ser lo que venía siendo" (Arciniegas, citado por Tünnermann, 1996, pp. 11-38).

Si bien hoy no se pone en duda la defensa de la autonomía universitaria, máximo legado de este movimiento estudiantil, dicho principio ha sido utilizado para servir a quienes persiguen intereses reaccionarios o distintos a las funciones sustantivas 
de la universidad. El cogobierno, otra de las máximas conquistas de este movimiento, ha conducido en ciertos casos a la politización de las decisiones académicas y al abuso del facilismo académico. La asistencia libre, reivindicación también fundamental de la Reforma, se hizo para abrir las puertas de la universidad a todos los sectores de la sociedad, pero en el largo plazo no logró este cometido. En cambio, en muchos casos no desarrolló una actitud disciplinaria y un compromiso con la docencia y la formación.

Hoy cuando los sistemas de acreditación por indicadores impulsados por los estados nacionales de América Latina se constituyen como un referente casi obligatorio para certificar la calidad educativa de las universidades -con base en un neologismo importado de la producción y de la experiencia de agencias privadas norteamericanas- con el propósito hacer más con menos recursos económicos, cabría preguntarse si acaso esta opción no es una nueva forma de lesión a la autonomía universitaria. Es muy cierto cuando Ángel Díaz Barriga (2010). Ilama la atención sobre el concepto líquido de la calidad educativa por responder a diversas interpretaciones, estar sometido a indicadores numéricos, desconocer el proceso formativo integral (margina lo sustantivo y lo simbólico) y homogeneizar las dinámicas institucionales (formatos rígidos, indicadores, chequeo de listas). ¿De qué autonomía universitaria podría hablarse hoy cuando la acreditación es impuesta por concepciones y experiencias foráneas y es muy cierto que las universidades han derivado a la empresarización (Aboites, 2008, p. 11) del clima institucional y hacia los mecanismos de control sobre el quehacer cotidiano?

A cien años de cumplirse la fecha conmemorativa de la Reforma de Córdoba su legado continúa vigente. El movimiento estudiantil de 1918 fue la respuesta al contexto particular de las cambiantes condiciones políticas y socioeconómicas de la nación Argentina y del orbe en aquel momento, pero también fue la expresión de una raíz americana en la cual el continente vivía la influencia del modernismo de Rubén Darío y su llamado a la unidad de los valores hispanoamericanos, del arielismo de Rodó y la reafirmación del ideal bolivariano de unidad de las repúblicas latinoamericanas.

Posterior a la Reforma y a estas corrientes de pensamiento que influyeron en ella, se han escrito millares de páginas sobre las repercusiones de Córdoba. Intelectuales dentro y fuera de América Latina fueron influenciados o se refirieron a este acontecimiento: Ingenieros, Kom, Vasconcelos, Alfredo Palacios, Haya de la Torre, Mariátegui, Mella, Ponce, Henríquez Ureña, Gabriela Mistral, Asturias, Arciniegas, Carlos Quijano, Ricaurte Soler, Unamuno y Waldo Frank, entre otros (Biagini, H., 2006, p. 287). Esta impronta o influencia de la Reforma tiene explicación no sólo por los principios originales ya referidos a la universidad, sino también por su concepción humanística (Biagini, H., 2006, p. 19). "Toda la educación -expresaron los estudiantes en 1918- es una larga obra de amor a los que aprenden (...) Si no existe una vinculación espiritual entre el que enseña y el que aprende, toda enseñanza es hostil y por consiguiente infecunda". 


\section{BIBLIOGRAFÍA}

Aboites, Hugo (2008). "Un libro indispensable”. En Tünnermann, Carlos. Noventa años de la Reforma Universitaria de Córdoba (1918-2008). Buenos Aires: Clacso.

(2010): El futuro de la Universidad en América Latina. Extraído el 5 de agosto de 2010 desde: http://www.riseu.unam.mx

Acevedo Tarazona, Álvaro (2006): "Reforma y movimiento universitario en América Latina: Siglo XX". En Lafuente Guantes, María Isabel, coordinadora. ¿Hacia dónde va la educación universitaria americana y europea? España: Universidad de León.

(2008): "Reforma y reformismo en la universidad de América Latina. De la propuesta de reforma de Andrés Bello al instrumentalismo reformista de los años sesentas y setentas del siglo XX". En Revista Historia y Espacio, junio de 2008.

Arciniegas, Germán, citado por Tünnermann, Carlos (1996): Breve historia del desarrollo de la universidad en América Latina. En La educación superior en el umbral del siglo XXI, Caracas: CRESALC.

Biagini, Hugo (2006): "Juventud e identidad: De la reforma universitaria al posmodernismo". En MARSISKE, Renate, coordinadora. Movimientos Estudiantiles en la historia de América Latina III, México: Universidad Autónoma de México, p. 287.

Biagini, Hugo (2006): "Movimientos Estudiantiles en América y Europa". En JUNTA PROVINCIAL DE HISTORIA DE CÓRDOBA, Junta Provincial de Córdoba, p. 19.

Buchbinder, Pablo (2005): Historia de las universidades argentinas. Buenos Aires: Sudamericana, p. 95.

Cantón, Darío; Moreno, José L. y Ciria, Alberto (2005): Argentina: la democracia constitucional y su crisis. Buenos Aires: Paidós.

Ceballos, Carlos (1986): Los estudiantes universitarios y la política (1955-1970). Buenos Aires: Centro Editor de América Latina, p. 42.

Ciria, Alberto y Sanguinetti Horacio (1987): La Reforma Universitaria /1 (19181983). Buenos Aires: Centro Editor de América Latina.

Del Pont, Luis Marco (2005): Historia del Movimiento Estudiantil Reformista. Córdoba: Universitas.

Díaz Barriga, Ángel (2010): La acreditación de programas (planes de estudio). Entre el formalismo y los procesos educativos. Extraído el 5 de agosto de 2010 desde: http://www.riseu.unam.mx 
La Gaceta Universitaria 1918-1919 (2009): Una mirada sobre el movimiento reformista en las universidades nacionales. Buenos Aires: Editorial Universitaria de Buenos Aires.

La Voz Del Interior. 2 de abril de 1919.

Manifiesto Liminar de la Reforma Universitaria. Recuperado el 18 de noviembre de 2009, de http://es.wikisource.org/wiki/Manifiesto Liminar

Marsiske, Renate (1999): "Clases medias, universidades y movimientos estudiantiles (1900-1950)". En Marsiske, Renate (coord.), Movimientos estudiantiles en la historia de América Latina, México, Centro de Estudios sobre la Universidad.

Sigal, Silvia (2002): Intelectuales y poder en Argentina: La década del sesenta. Argentina: Siglo XXI.

Tünnermann, Carlos (1983): La Reforma Universitaria de Córdoba. Caracas: FEDES.

-------- (2008): Noventa años de la Reforma Universitaria de Córdoba (19182008), Buenos Aires: Clacso.

VERA, Cristina (s.f.): "Reformas y contrarreformas de la Universidad de Córdoba", en Soto Arango, Diana; Lucena Salmoral, Manuel y Rincón, Carlos, directores. Estudios sobre la universidad latinoamericana: De la colonia al siglo XXI. Tunja: Rudecolombia.

Recibido: Noviembre 23 de 2010

Aprobado: Mayo 9 de 2011 